\title{
Behavioral management of night eating disorders
}

This article was published in the following Dove Press journal:

Psychology Research and Behavior Management

25 March 2013

Number of times this article has been viewed

Laura A Berner'

Kelly C Allison ${ }^{2}$

'Department of Psychology, Drexel University, ${ }^{2}$ Department of Psychiatry, Perelman School of Medicine, University of Pennsylvania,

Philadelphia, PA, USA
Correspondence: Kelly C Allison Perelman School of Medicine, University of Pennsylvania, 3535 Market St, Suite 3027, Philadelphia, PA 19104, USA

Tel +l 2158987314

Email kca@mail.med.upenn.edu
Abstract: Night eating syndrome (NES) is a form of disordered eating associated with evening hyperphagia (overeating at night) and nocturnal ingestions (waking at night to eat). As with other forms of disordered eating, cognitive and behavioral treatment modalities may be effective in reducing NES symptoms. This review presents evidence for a variety of behavioral treatment approaches, including behavioral therapy, phototherapy, behavioral weight loss treatment, and cognitive-behavioral therapy. A more detailed overview of cognitive-behavioral therapy for NES is provided. All of these studies have been case studies or included small samples, and all but one have been uncontrolled, but the outcomes of many of these approaches are promising. Larger randomized controlled trials are warranted to advance NES treatment literature. With the inclusion of NES in the fifth edition of the Diagnostic and Statistical Manual of Mental Disorders (DSM-5) as a "Feeding or Eating Disorder Not Elsewhere Classified," more sophisticated, empirically-supported, behaviorally-based treatment approaches are much needed.

Keywords: night eating syndrome, cognitive-behavioral treatment, phototherapy, behavioral weight loss, behavior therapy

\section{Night eating syndrome (NES): definition and diagnosis}

The diagnostic criteria for NES have evolved since its first description in $1955 .{ }^{1}$ Varying operational definitions of diagnostic criteria for NES and inconsistent assessment measures used in NES research studies ${ }^{2,3}$ have complicated cross-study comparison and slowed advances in understanding NES. Though NES was not formally included in previous versions of the Diagnostic and Statistical Manual of Mental Disorders, for the first time, in the fifth edition of the Diagnostic and Statistical Manual of Mental Disorders (DSM-5), the proposed diagnostic criteria for NES are described under Feeding or Eating Disorders Not Elsewhere Classified. These criteria include: recurrent episodes of night eating, including nocturnal ingestions characterized by waking from sleep and eating or excessive consumption of food after the evening meal; awareness and recall of night eating; and significant distress or functional impairment associated with the night eating. The night eating cannot be better accounted for by changes in the sleep cycle, cultural norms, binge eating disorder (BED), another psychiatric disorder, substance abuse or dependence, a general medical disorder, or medication side effects. ${ }^{4}$ While differential diagnosis should be made carefully, depression and anxiety are often comorbid with NES, and their presence does not automatically preclude a diagnosis of NES. Before these DSM-5 criteria were drafted, an international meeting was held in April 2008 to develop a consensus for proposed diagnostic criteria for NES. ${ }^{5}$ This description conceptualized submit your manuscript $\mid$ www.dovepress.com

Dovepress

http://dx.doi.org// 0.2147/PRBM.S31929
Psychology Research and Behavior Management 2013:6 I-8

(C) 2013 Berner and Allison, publisher and licensee Dove Medical Press Ltd. This is an Open Access article which permits unrestricted noncommercial use, provided the original work is properly cited. 
NES as a disorder of delayed circadian intake of food and offered more guidance on criterion requirements; for example, the proposed criteria from the 2008 consensus meeting detail that for "recurrent, excessive evening intake," at least $25 \%$ of food intake would have to be consumed after the evening meal, on average, for at least 3 months. ${ }^{5}$ These criteria also list five qualifiers, three of which would be required for diagnosis. These include morning anorexia, a strong desire to eat in the evening or nighttime, sleep onset or maintenance problems, the belief that one needs to eat to fall asleep, and depressed mood that often worsens throughout the day. These must be present at least half the time. Additionally, the 2008 proposed criteria specified that nocturnal ingestions would be considered clinically significant if they occurred twice per week for at least 3 months.

The authors of these previously drafted criteria clarified that NES could be diagnosed co-morbidly with another eating disorder. ${ }^{5}$ In fact, research suggests that among individuals with NES, approximately $7 \%-25 \%$ also met criteria for BED as described in the fourth edition, text revision of the DSM $(D S M-I V-T R),{ }^{6-9}$ and approximately $9 \%$ of individuals with bulimia nervosa also have NES. ${ }^{6}$ These rates of diagnostic overlap are consistent with those recently reported in a large Swiss sample of young adults aged 18-26 years. ${ }^{10}$

Validated self-report measures and semi-structured interviews have been developed for the assessment of NES symptoms, including the Night Eating Questionnaire (NEQ) - a self-report measure, ${ }^{11}$ the NES History and Inventory - a diagnostic interview, ${ }^{12}$ and the Night Eating Symptom Scale - a self-report measure based on the NEQ used during treatment to assess symptom change in the previous week. ${ }^{12}$ In addition, a single item of the Eating Disorder Examination ${ }^{13}$ - a semi-structured interview that is considered the "gold standard" in eating disorder assessment - assesses for the presence of nocturnal ingestions.

\section{Method}

The present summary of the literature does not represent a systematic review. To identify potential studies for inclusion, the keywords "night eating syndrome treatment" and "night eating syndrome behavioral" were used to search the PubMed, MedView, and PsycINFO databases. Additional articles and chapters from the reference lists of database search results were also included.

\section{NES treatment: pharmacological interventions and phototherapy}

Despite inconsistent definitions of NES, several trials have investigated treatments for NES symptoms. Early treatment approaches to NES symptoms among obese individuals were psychodynamically oriented and focused on stress reduction. ${ }^{14}$ Pharmacotherapy trials have since dominated the treatment of NES literature. One case series of paroxetine or fluvoxamine, ${ }^{15}$ two open-label trials of sertraline, ${ }^{16,17}$ and one randomized, placebo-controlled trial of sertraline ${ }^{18}$ indicated successful treatment of NES with selective serotonin reuptake inhibitors (SSRIs). The results of a 12-week, randomized, placebo-controlled trial of escitalopram (another SSRI) indicated NES symptom improvement with the active medication, but these improvements were not statistically significantly different from those found in the placebo group. ${ }^{19}$

Phototherapy, or exposure to certain wavelengths of light via a light-emitting device for prescribed amounts of time, has been investigated for the treatment of mood disorders and sleep disorders. The effect of phototherapy on melatonin, a key regulator of circadian rhythm, prompted trials of phototherapy as a potential treatment for NES, which has been conceptualized by some as a disorder of delayed circadian rhythm. Phototherapy has shown to be effective for NES in two case studies. The first case, an obese woman with nonseasonal major depressive disorder who was also taking paroxetine, remitted from NES after 14 days of daily phototherapy. ${ }^{20}$ This case study followed a "BAB" design in which a no-treatment phase (A phase) followed the initial delivery of treatment (B phase), followed by the reintroduction to treatment (second B phase). It was reported that the client became symptomatic after discontinuation of phototherapy, and remitted once more after 12 additional days of phototherapy. The second case, a normal-weight male, remitted from both nonseasonal major depressive disorder and NES following 14 sessions of daily phototherapy. ${ }^{21}$ Although these very preliminary findings are promising for individuals with NES and nonseasonal depression, randomized controlled trials are needed to confirm the efficacy of phototherapy for the treatment of NES.

\section{NES treatment: behavioral approaches}

Two early case studies indicated improvement in night eating symptoms following behavioral interventions. A case study of a 30-year-old male detailed response prevention strategies, including placing signs on the client's refrigerator, positioning a chair in his hallway between his bedroom and kitchen to awaken him, discontinuing eating while standing next to the refrigerator, placing kitchen knives in the client's car, and ultimately locking his refrigerator with a chain. ${ }^{22}$ These strategies reduced night eating episodes, but urges to 
eat at night persisted at 18-month follow-up. A 24-year-old female with bulimia nervosa, rumination, and night eating that was characterized by nocturnal ingestions after sleeping 1-3 hours was the subject of a second case study. ${ }^{23}$ Treatment of night eating involved a contingency management strategy of rewarding the client with the return of her jewelry (held by the treatment provider at the start of therapy) with abstinence. The client also attended a cognitive-behavioral group for the treatment of bulimia nervosa. This combined individual and group cognitive-behavioral treatment (CBT) resulted in elimination of night eating behaviors, although the element of the treatment that contributed most to this change is unknown.

Since these initial case studies, two main behavioral treatments have been investigated: progressive muscle relaxation and behavioral weight loss. Only one controlled behavioral trial of progressive muscle relaxation for NES has been published. Based on the findings of high levels of stress associated with NES, this study compared a group who received a behavioral stress management intervention - a 1-week abbreviated progressive muscle relaxation therapy (APMRT; 20 minutes per night) - to a control group who quietly sat for a matched amount of time. ${ }^{24}$ The APMRT group reported significantly decreased evening appetite and increased morning appetite, in addition to lower levels of stress, fatigue, and anxiety. Decreases in nocturnal ingestion intake and increases in breakfast intake were not statistically significant, but effect sizes for these differences were large. Results from this brief, small trial indicate that further investigation of the efficacy of progressive muscle relaxation for NES symptoms is warranted.

Two notable investigations have applied behavioral weight loss strategies to individuals with NES. Gluck et al investigated the effects of a medically-supervised liquid diet in individuals with and without NES, and reported that after 1 month, those with NES lost less weight. ${ }^{25}$ They did not investigate, however, the effects of this intervention on symptoms of NES.

Dalle Grave et al conducted a more recent investigation of a 21-day inpatient treatment in obese individuals with and without NES followed by either outpatient treatment with an obesity specialist in $62 \%$ of the sample or no treatment in the remaining $48 \%{ }^{26}$ Equal proportions of night eaters received ongoing care after discharge. The inpatient program included a low-calorie diet and a regular pattern of eating, daily exercise, and psychoeducational group therapy focusing on behavioral weight loss strategies. This intervention resulted in body mass index reduction across all individuals: those with NES $(\mathrm{n}=32) \operatorname{lost} 1.9 \mathrm{~kg} / \mathrm{m}^{2}$ on average, while those without NES $(n=68) \operatorname{lost} 1.5 \mathrm{~kg} / \mathrm{m}^{2}$ in the inpatient treatment. However, these reductions in body mass index were not statistically significantly different. Similarly, at 6-month follow-up, the percent of weight loss from baseline weight among those with NES (6.4\%) and those without NES (8.2\%) did not differ. It was reported that there were no differences in body mass index reduction at 6 months for those who continued night eating as opposed to those who had discontinued these behaviors. Although behavioral weight loss did not have a differential impact on weight loss among those with and without NES, only $27.6 \%$ of individuals who originally met criteria for NES retained the diagnosis at the 6-month follow-up. In addition, 62.1\% of those with NES at baseline reported that they had been abstinent from night eating for 3 months.

The results of this study suggest that the behavioral weight loss treatment was effective in controlling night eating symptoms for a large proportion of those presenting with an NES diagnosis. Behavioral weight loss treatment has also been shown to reduce both binge eating episode frequency and weight in clients with $\mathrm{BED},{ }^{27}$ suggesting that the behavior modification approaches contained in structured behavioral weight loss programs impact disordered eating behaviors that are related to overweight and obesity, such as NES and BED. Although the active ingredients of behavioral weight loss key to reducing night eating symptoms cannot be identified in the investigation of Dalle Grave et al, two elements of the treatment seem likely candidates. First, the added structure to food intake during the day in behavioral weight loss may be an important factor in modifying the delayed pattern of eating that is characteristic of NES. Indeed, Boston et al have shown that individuals with NES report unscheduled, inconsistent mealtimes over the 24-hour day as compared to control participants, and this may drive night eating behaviors. ${ }^{28}$ Self-monitoring of food intake is the second likely key element of behavioral weight loss approaches in the treatment of NES. Just as self-monitoring is generally a strong predictor of successful weight loss, ${ }^{29}$ it may also promote increased awareness of the impact of night eating episodes on weight and may help clients reevaluate their drive to eat and observe patterns in their eating behaviors.

\section{CBT for NES \\ Theoretical basis and treatment techniques}

The success of several behavior-focused interventions for NES suggests that contingency management is an essential 
element of NES treatment; however, conflict between the normal boundaries of sleep and the abnormal schedule of food intake characteristic of NES often contributes to the development of faulty cognitions about the function of eating at night or nocturnal ingestions. ${ }^{30}$ These include, for example, a belief that one needs to eat to resume sleep, thoughts about being incapable of avoiding eating at night, and thoughts about needing to eat to alleviate anxiety or agitation in the evening. Of note, this belief in the need to eat to fall asleep seems to represent an important differential diagnostic indicator between BED and NES, and possibly insomnia and NES.

CBT for NES therefore integrates behavioral interventions with cognitive techniques standard in CBT aimed at addressing these seemingly integral distorted thoughts. This includes the use of dysfunctional thought records, developed by Beck et $\mathrm{al}^{31}$ in which clients record objective situations, resulting thoughts, emotions, and behavioral outcomes. This examination of distorted thoughts is complemented by stimulus control interventions in CBT for NES to help clients test the validity of their thoughts (eg, of the inevitability of their night eating or their inability to resume sleep without eating) via behavioral experimentation. This identification of underlying automatic thoughts and the examination of environmental cues and emotions associated with night eating permit functional analysis of night eating behaviors. Other standard elements of CBT as conceived by Beck et al, including Socratic questioning, the "downward arrow" technique to identify core beliefs, and collaborative empiricism are employed.

Elements of CBT for insomnia (CBT-I) - which has been shown to be highly effective in the treatment of insomnia also served as a theoretical framework for the development of CBT for NES. Components of CBT-I, such as improving sleep hygiene and standardizing bedtime and morning awakening times, ${ }^{32}$ were added as important elements to CBT for NES.

As is typical in most psychotherapeutic interventions, elements of rapport building and early investment in the therapeutic alliance are essential to CBT for NES. These more basic aspects of treatment may be particularly critical, as the workload of this therapy is high, and readiness for such involvement on the part of the client seems crucial for preventing treatment dropout.

\section{CBT for NES: treatment overview}

Before beginning treatment, a structured assessment of current NES symptoms is recommended. This may include the use of validated NES measures such as the NEQ - a self-report screening tool, ${ }^{11}$ and the NES History and Inventory - a diagnostic interview. ${ }^{12}$ In addition, assessment of potential comorbid disorders that would trump the treatment of NES, including severe depression, suicidal ideation, and anxiety disorders, should be completed before initiation of CBT for NES.

CBT for NES occurs in three basic stages, and consists of ten 1-hour sessions. ${ }^{33}$ These sessions initially occur on a weekly basis, and the final two sessions are scheduled for every other week. During the first stage of treatment, the therapist focuses on development of rapport with the client, psychoeducation about night eating, and explanation of the CBT rationale. Client homework includes self-monitoring of behaviors, completion of thought records, and behavioral experimentation. When night eating episodes occur, behavioral chain analyses are conducted, first collaboratively with the therapist in session and then for homework, to help identify cognitive and behavioral intervention targets. Assignments for decreasing nocturnal ingestions could include placing signs on doors, in the bathroom, and on the refrigerator with statements meaningful to the client that would help him remember his daytime intentions for not eating at night, thereby disrupting the automation of the typical nighttime eating routine. Other strategies could include placing barriers along the path to the kitchen, removing food or locking cabinets to limit access to preferred night-eating foods, and completing self-monitoring forms to raise awareness and identify intervention targets. ${ }^{33}$ As similar stimulus control and structured eating pattern interventions are shared by this CBT for NES and by standard behavioral weight loss treatment, weight loss is included as a goal for those wishing to reduce their weight. For these individuals, caloric monitoring is included with self-monitoring of night eating behaviors and sleep patterns (time of sleep onset, time and duration of nocturnal awakenings, and morning rise time).

In the second stage of treatment, the therapist and client work collaboratively to identify thematic patterns in self-monitoring that are used to tailor treatment. Cognitive restructuring is taught and practiced, and clients engage in behavioral experiments and stimulus control to challenge automatic thoughts as they are identified. Breakfast is also added to the client's pattern of eating. For clients with comorbid major depression, cognitive distortions beyond the bounds of night eating are also addressed in this second stage of treatment, as these automatic thoughts, along with those related to anxiety or stress, may serve as catalysts for night eating..$^{30}$ If appropriate, alternative strategies to night 
eating, including progressive muscle relaxation and deep breathing exercises, are introduced in these sessions. Sleep hygiene and physical activity are also addressed in this stage, as these elements are thought to promote sounder sleep and improve weight management.

For the final two sessions, which comprise the third stage of CBT for NES, treatment transitions to biweekly sessions. These sessions include summarizing and reflecting upon progress and bolstering the client's confidence and sense of self-efficacy to continue on his or her own with successful changes. These final sessions also focus on relapse prevention through prediction of future challenges and anticipatory problem solving.

\section{Evidence in support of CBT for NES}

To date, only one empirical investigation of CBT for NES has been published. This pilot study of the ten-session CBT for NES protocol, described above, included 25 individuals with NES. ${ }^{34}$ After treatment, the number of nocturnal ingestions decreased significantly as did calories from nocturnal ingestions (from 8.7 to 2.6 per week). There was a statistically non significant decrease, from $35 \%$ to $24.9 \%$, in the proportion of calories consumed after dinner. Total daily intake, number of awakenings, and Night Eating Symptom Scale total scores all decreased significantly. Participants lost a significant amount of weight, as this was also a target of treatment for most clients, and depression scores were reduced. With regard to components of night eating, the amount of food consumed during nocturnal ingestions was reduced significantly, but the quantity consumed before bedtime was not significantly affected.

From the perspective of clinical significance, the percentage of intake consumed after dinner decreased after treatment to a level just below the diagnostic boundary for NES. This effect is largely attributable to the reduction in nocturnal ingestions. Compared to prior investigations, outcome results are similar to those of the aforementioned sertraline trial. ${ }^{16}$ The results also suggest that CBT improves outcome compared to progressive muscle relaxation alone. ${ }^{24}$

\section{The impact of weight status on night eating symptom expression and treatment response}

In addition to consideration of comorbidities in treatment planning for individuals with NES, research indicates that weight status represents an important variable in both the expression of night eating symptoms and in response to NES treatment. Marshall et al were the first to show that compared to obese individuals with NES, individuals at a normal body weight with NES reported more severe nocturnal eating symptoms, including more awakenings, more cravings when up at night, and more nocturnal ingestions, as measured by the NEQ. ${ }^{35}$ Subsequently, Lundgren et al further characterized normal-weight individuals with NES $(\mathrm{N}=19)$, reporting that they consumed $50 \%$ of their daily caloric intake after dinner and experienced 13 awakenings (getting up out of bed) and ten nocturnal ingestions per week, on average. ${ }^{36}$ These figures are higher than those reported among overweight and obese individuals with NES, who have been reported in one sample $(\mathrm{N}=46)$ to consume $35 \%$ of their intake after dinner and experience approximately eleven awakenings and eight nocturnal ingestions per week. ${ }^{37}$

Findings that normal-weight individuals with NES eat a higher percentage of their intake at night than overweight and obese sufferers may seem counterintuitive. One potential explanation is that individuals of normal weight with NES more actively restrict their intake during the day and exercise to counteract their nightly caloric intake. This may result in the first meal of the day occurring much later, delaying food intake even more severely than that of overweight and obese persons with NES, who report eating more calories generally throughout the day. Increased dietary restriction during the day in the normal-weight group may therefore exert an increased biological pressure to eat, which may, in turn, trigger more frequent nocturnal ingestions, thereby reinforcing the night-eating cycle.

Treatment recommendations for individuals with NES who are normal-weight may also differ from those for overweight and obese clients with NES. Because of the more extreme pattern that includes dietary restriction during the day, normalweight clients with NES may fear weight gain as a result of NES treatment. This may preclude normal-weight clients from adopting a pattern of eating that includes earlier meals for fear that they will be eating both all day and all night, thus interfering with treatment adherence. In fact, as shown in the investigation of Allison et al, ${ }^{34}$ this was not the case; normal-weight participants did not gain weight during this pilot study of CBT. Of note, behavioral weight loss components, including calorie counting, were omitted from the CBT treatment of normal-weight participants. Interventions with normal-weight individuals with NES may require increased focus on weight-gain related fears. Further, as shown in Figure 1, normal-weight participants have been found to demonstrate reductions in percentage of food intake after dinner and their number of nocturnal ingestions per week at a similar rate as overweight and obese participants receiving CBT for NES, but they ended with higher symptom 

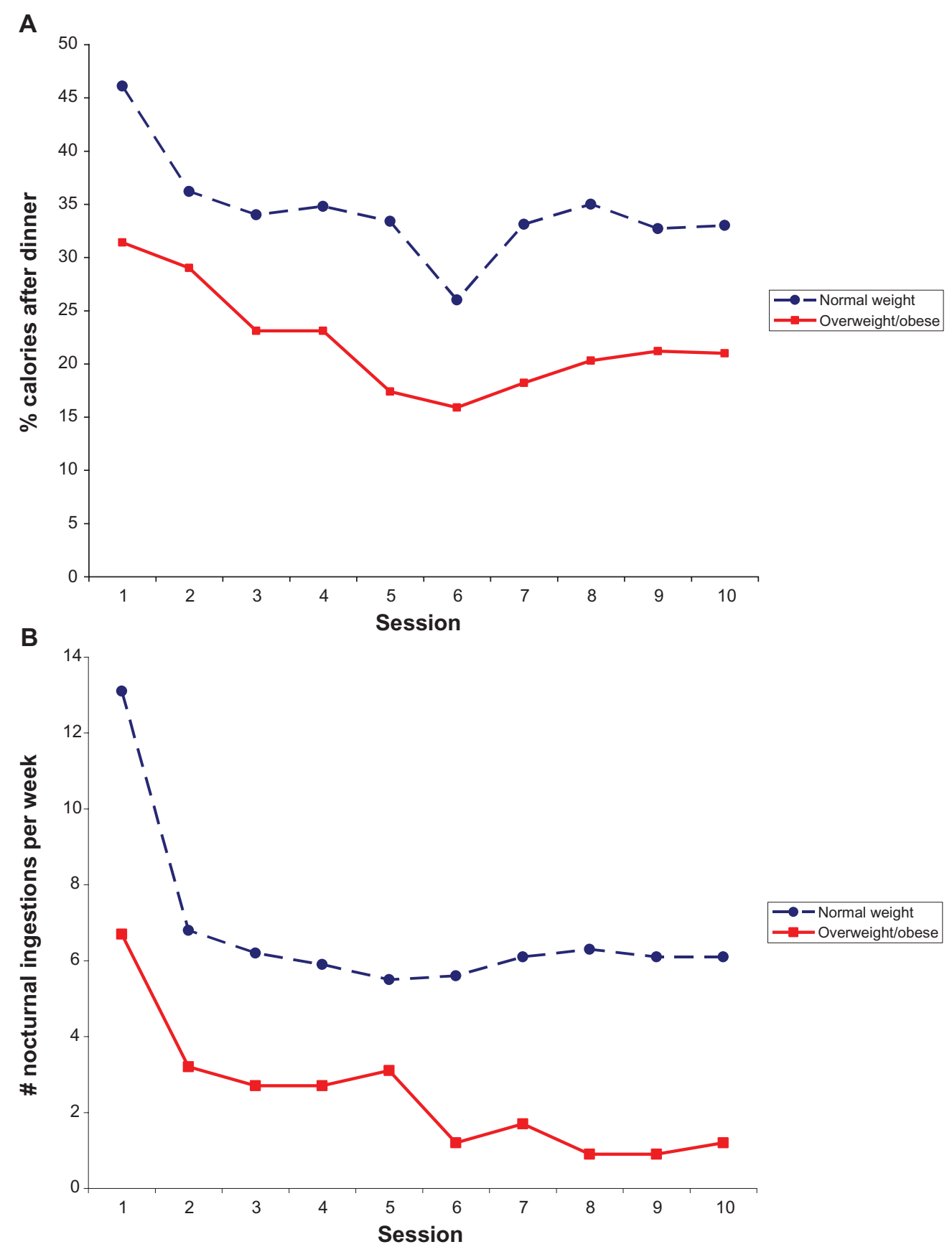

Figure I (A) Change in proportion of calories consumed after the evening meal between normal-weight $(n=8)$ and overweight/obese participants $(n=I 7)$. The proportion of calories decreased over time in both groups $(F[10,139]=5.23, P<0.000 I)$ and the levels of caloric intake differed between the weight groups $(F[1,23]=6.16, P=0.02)$. (B) Nocturnal ingestions per week decreased significantly and at similar rates for both weight groups $(F[9,123]=8.23, P<0.000 I)$.

Used with permission of the Association for the Advancement of Psychotherapy. Allison KC, Lundgren JD, Moore RH, O'Reardon JP, Stunkard AJ. Cognitive behavior therapy for night eating syndrome: a pilot study. Am J Psychother. 2010;64(I):91-106. ${ }^{34}$

levels as a result of this baseline difference. Longer-term treatment for this normal-weight group may be warranted, given the higher level of symptom severity.

\section{Future research directions}

Non-standardized and frequently changing diagnostic definitions of NES have challenged the study of the treatment of the disorder. Initial investigations of SSRIs, phototherapy, behavioral, and cognitive-behavioral interventions for NES have all demonstrated some degree of success in reducing or eliminating night eating. Despite promising evidence from initial trials, further research investigating efficacious treatments for NES is needed. Randomized controlled trials of CBT for NES are needed. Further, studies that include larger sample sizes of individuals who are normal weight, overweight, and obese are necessary to parse the relative benefits of CBT 
for NES across weight classes. In addition to randomized controlled studies comparing CBT with other treatment modalities, dismantling studies seem warranted to identify the active ingredients in this treatment. Extant evidence suggests that ten sessions produce promising outcomes; however, longer durations of treatment should be tested, as there have been no long-term studies examining the durability of this treatment generally, or duration of remission in treatment responders. Finally, the effect of psychiatric comorbidities on treatment outcome is unknown, and because of the high rate of overlap between NES and other eating, mood, anxiety, and substance use disorders, structured investigations of the impact of comorbidity on treatment response are needed.

\section{Disclosure}

The authors report no conflicts of interest in this work.

\section{References}

1. Stunkard AJ, Grace WJ, Wolff HG. The night-eating syndrome: a pattern of food intake among certain obese patients. Am J Med. 1955;19(1): 78-86.

2. Striegel-Moore RH, Franko DL, Garcia J. The validity and clinical utility of night eating syndrome. Int J Eat Disord. 2009;42(8):720-738.

3. Striegel-Moore RH, Franko DL, May A, Ach E, Thompson D, Hook JM. Should night eating syndrome be included in the DSM? Int J Eat Disord. 2006;39(7):544-549.

4. DSM-5 development [webpage on the Internet]. Arlington, VA: American Psychiatric Association; 2012. Available from: http://www dsm5.org/ProposedRevision/Pages/proposedrevision.aspx?rid=26. Accessed February 7, 2013.

5. Allison KC, Lundgren JD, O'Reardon JP, et al. Proposed diagnostic criteria for night eating syndrome. Int J Eat Disord. 2010;43(3):241-247.

6. Allison KC, Grilo CM, Masheb RM, Stunkard AJ. Binge eating disorder and night eating syndrome: a comparative study of disordered eating. J Consult Clin Psychol. 2005;73(6):1107-1115.

7. Tzischinsky O, Latzer Y. Nocturnal eating: prevalence, features and night sleep among binge eating disorders and bulimia nervosa patients in Israel. Eur Eat Disord Rev. 2004;12(2):101-109.

8. Stunkard AJ, Berkowitz R, Wadden T, Tanrikut C, Reiss E, Young L. Binge eating disorder and the night-eating syndrome. Int J Obes Relat Metab Disord. 1996;20(1):1-6.

9. Greeno CG, Wing RR, Marcus MD. Nocturnal eating in binge eating disorder and matched-weight controls. Int J Eat Disord. 1995;18(4):343-349.

10. Fischer S, Meyer AH, Hermann E, Tuch A, Munsch S. Night eating syndrome in young adults: Delineation from other eating disorders and clinical significance. Psychiatry Res. 2012;200(2-3):494-501.

11. Allison KC, Lundgren JD, O'Reardon JP, et al. The Night Eating Questionnaire (NEQ): psychometric properties of a measure of severity of the night eating syndrome. Eat Behav. 2008;9(1):62-72.

12. Lundgren JD, Allison KC, Vinai P, Gluck ME. Assessment instruments for night eating syndrome. In: Lundgren JD, Allison KC, Stunkard AJ, editors. Night Eating Syndrome: Research, Assessment, and Treatment. New York, NY: Guilford Press; 2012:197-220.

13. Fairburn CG, Cooper Z, O'Connor ME. Eating Disorder Examination (Edition 16.0D). In: Fairburn CG, editor. Cognitive Behavior Therapy and Eating Disorders. New York, NY: Guilford Press; 2008:265-309.

14. Stunkard AJ. The Pain of Obesity. Palo Alto, CA: Bull Publishing; 1976.

15. Miyaoka T, Yasukawa R, Tsubouchi K, et al. Successful treatment of nocturnal eating/drinking syndrome with selective serotonin reuptake inhibitors. Int Clin Psychopharmacol. 2003;18(3):175-177.
16. O'Reardon JP, Stunkard AJ, Allison KC. Clinical trial of sertraline in the treatment of night eating syndrome. Int J Eat Disord. 2004;35(1): 16-26.

17. Stunkard AJ, Allison KC, Lundgren JD, et al. A paradigm for facilitating pharmacotherapy at a distance: sertraline treatment of the night eating syndrome. J Clin Psychiatry. 2006;67(10):1568-1572.

18. O'Reardon JP,Allison KC, Martino NS, Lundgren JD, Heo M, StunkardAJ. A randomized, placebo-controlled trial of sertraline in the treatment of night eating syndrome. Am J Psychiatry. 2006;163(5): 893-898.

19. Vander Wal JS, Gang CH, Griffing GT, Gadde KM. Escitalopram for treatment of night eating syndrome: a 12-week, randomized, placebocontrolled trial. J Clin Psychopharmacol. 2012;32(3):341-345.

20. Friedman S, Even C, Dardennes R, Guelfi JD. Light therapy, obesity, and night-eating syndrome. Am J Psychiatry. 2002;159(5):875-876.

21. Friedman S, Even C, Dardennes R, Guelfi JD. Light therapy, nonseasonal depression, and night eating syndrome. Can J Psychiatry. 2004; 49(11):790.

22. Coates TJ. Successive self-management strategies towards coping with night eating. J Behav Ther Exp Psychiatry. 1978;9(2):181-183.

23. Williamson DA, Lawson OD, Bennett SM, Hinz L. Behavioral treatment of night bingeing and rumination in an adult case of bulimia nervosa. J Behav Ther Exp Psychiatry. 1989;20(1):73-77.

24. Pawlow LA, O'Neil PM, Malcolm RJ. Night eating syndrome: effects of brief relaxation training on stress, mood, hunger, and eating patterns. Int J Obes Relat Metab Disord. 2003;27(8):970-978.

25. Gluck ME, Geliebter A, Satov T. Night eating syndrome is associated with depression, low self-esteem, reduced daytime hunger, and less weight loss in obese outpatients. Obesity. 2001;9(4):264-267.

26. Dalle Grave R, Calugi S, Ruocco A, Marchesini G. Night eating syndrome and weight loss outcome in obese patients. Int J Eat Disord. 2011;44(2):150-156.

27. Munsch S, Meyer AH, Biedert E. Efficacy and predictors of long-term treatment success for cognitive-behavioral treatment and behavioral weight-loss-treatment in overweight individuals with binge eating disorder. Behav Res Ther. 2012;50(12):775-785.

28. Boston RC, Moate PJ, Allison KC, Lundgren JD, Stunkard AJ. Modeling circadian rhythms of food intake by means of parametric deconvolution: results from studies of the night eating syndrome. Am J Clin Nutr. 2008;87(6):1672-1677.

29. Wilde MH, Garvin S. A concept analysis of self-monitoring. JAdv Nurs. 2007;57(3):339-350.

30. Allison KC, Stunkard AJ, Thier SL. Overcoming Night Eating Syndrome: A Step-By-Step Guide to Breaking the Cycle. Oakland, CA: New Harbinger Publications; 2004.

31. Beck AT, Rush AJ, Shaw BF, Emery G. Cognitive Therapy of Depression. New York, NY: Guilford Press; 1979.

32. Perlis ML, Smith MT, Jungquist C, Nowakowski S, Orff H, Soeffing J. Cognitive-behavioral therapy for insomnia. In: Attarian HP, Schuman C, editors. Clinical Handbook of Insomnia, 2nd ed. New York, NY: Humana Press; 2010:281-296.

33. Allison KC. Cognitive-behavioral therapy manual for night eating syndrome. In: Lundgren JD, Allison KC, Stunkard AJ, editors. Night Eating Syndrome: Research, Assessment, and Treatment. New York, NY: Guilford Press; 2012:246-265.

34. Allison KC, Lundgren JD, Moore RH, O'Reardon JP, Stunkard AJ. Cognitive behavior therapy for night eating syndrome: a pilot study. Am J Psychother. 2010;64(1):91-106.

35. Marshall HM, Allison KC, O'Reardon JP, Birketvedt G, Stunkard AJ. Night eating syndrome among nonobese persons. Int J Eat Disord. 2004;35(2):217-222.

36. Lundgren JD, Allison KC, O'Reardon JP, Stunkard AJ. A descriptive study of non-obese persons with night eating syndrome and a weightmatched comparison group. Eat Behav. 2008;9(3):343-351.

37. O'Reardon JP, Ringel BL, Dinges DF, et al. Circadian eating and sleeping patterns in the night eating syndrome. Obes Res. 2004;12(11): 1789-1796. 


\section{Publish your work in this journal}

Psychology Research and Behavior Management is an international, peerreviewed, open access journal focusing on the science of psychology and its application in behavior management to develop improved outcomes in the clinical, educational, sports and business arenas. Specific topics covered include: Neuroscience, memory \& decision making; Behavior modification \& management; Clinical applications; Business \& sports performance management; Social and developmental studies; Anima studies. The manuscript management system is completely online and includes a quick and fair peer-review system. Visit http://www.dovepress. com/testimonials.php to read real quotes from published authors.

Submit your manuscript here: http://www.dovepress.com/psychology-research-and-behavior-management-journal 